\title{
Bond-Graph model based stepper motor output characteristics simulation implemented in LabVIEW environment
}

\author{
Zsolt Molnár \\ Mechatronics Department \\ University of Debrecen \\ Debrecen, Hungary \\ molnar.zsolt@eng.unideb.hu
}

\author{
Géza Husi \\ Mechatronics Department \\ University of Debrecen \\ Debrecen, Hungary \\ husigeza@eng.unideb.hu
}

\begin{abstract}
In the industry, simulations are of great importance. They enable measurements to be made in different conditions about a virtual device, which are highly comparable to measurements made in real-life scenarios.

Because of their wide range of usage in lower power drive systems, where precision and simplicity is a must, the subject of study is a permanent magnet stepper motor. For precise positioning purposes, it is essential to know the positioning behaviour of these devices. The model construction process involved using a pre-defined Bond-Graph of the stepper motor available in the literature in this field.
\end{abstract}

In the next step, the Bond-Graph model was converted to a block diagram of the motor. For simulations purposes, the LabVIEW visual programming environment was chosen. LabVIEW enables the direct implementation of block diagrams and simulating their operation.

The preliminary steps allows us to check and confirm the functionality and correctness of the model.

This article covers in detail the model conversion and implementation steps of the simulation. At the end, the functionality of the simulation was tested.

Keywords-stepper motor; Bond-graph; simulation; LabVIEW; block diagram

\section{INTRODUCTION}

In the Mechatronics Department, at the University of Debrecen, there are many projects involving different types of motors. A general categorization of these motors and the devices that use them are presented. The review gives an insight into the ongoing projects at the Department.

In the Robotics Laboratory, the KUKA KR5 Arc robot [1] it contains an array of servomotors. Servomotors are special kind of motors, which have position feedback. Therefore they can be positioned precisely, and can detect potential mechanical overloads, making them suitable for robot arms.

Another category of motors are the brushless DC motors, used for electrically driven vehicles, especially for UAVs. The simulation of a quadcopter driven by brushless DC motors was realized during one of the research projects at the Department [2].

Another category of motors, used for precise positioning, beside the servo motors, are the stepper motors. These are a kind of motors which require driver circuitry in order to rotate. In comparison with servo motors, there are much more cost-efficient, and the driver circuitry is also simpler [3]. For these reasons they are used in smaller projects, like 3D printers, plotters and any other project, were high torque and position feedback is not a must.

During the modelling and the simulation process, the following topics will be covered: defining the physical characteristics of the motor (size, state variables, operating principle), the Bond-graph of the motor, the mathematical model and finally the implementation of the simulation in LabVIEW.

\section{Physical Characteristics OF THE MOtoR}

The subject of study is a permanent magnet stepper motor used in a bipolar configuration. The permanent magnet type and bipolar setup was chosen because of their ubiquity both in precision and small scale mechanisms and in low cost, high reliability, lower power actuators, also used by the automotive industry. Besides these, another advantage of the permanent magnet stepper motors is that they can be manufactured in small form factor (compared to the hybrid stepper motors) and deliver relatively high torque at the same time. In contrast with the variable-reluctance stepper motors, the permanent magnet type provide a detent torque. This is the ability of the motor to exert a certain amount of position holding torque in an unpowered state.

The bipolar configuration means, that the motor has two separated coils. These coils are powered by two polarity inverting circuits, known as H-bridges [4].

The studied motor type is 35PM24L01, PM Stepper motor with 15 degrees step angle [5]. Calculated from step angle, it takes 24 steps for the motor to complete a 360 degree rotation. 35PM24L01 motor was chosen, because it has all the parameters specified by the manufacturer, which are required for the 
simulation. Furthermore the motor has a low commercial value, so multiple motors can be purchased at low price, for further, real-life measurements. These results are usable for simulation model validation. The most important dimensions are presented on 1. Figure.

The chosen stepper motor is an 35PM24L01, which has the following characteristics [5]:

- $\quad$ Current per winding: $0.8 \mathrm{~A}$

- Holding torque: $0.0539 \mathrm{Nm}$

- Detent torque: $0.0147 \mathrm{Nm}$

- Resistance per winding: $15 \Omega$

- Inductance per winding: $13.5 \mathrm{mH}$

- Rotor inertia: $0.00000075 \mathrm{kgm}^{2}$

- Pole pair number: 3

- Step angle: $15^{\circ}(0.261799 \mathrm{rad})$

- Weight: $86.6 \mathrm{~g}$

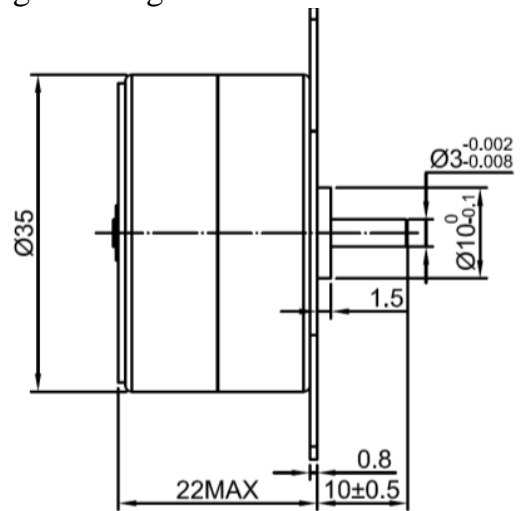

1. Figure: Side view of the motor-dimensions are in [mm] [5]

\section{PHYSICAL MODELING}

The stepper motors are classified in three categories according to its physical characteristics: reluctance-, permanent magnet and hybrid stepper motors [6]. They have stator phases wound on stator saliencies.

The variable-reluctance stepper motor has a rotor with saliencies, without permanent magnetization.

The permanent magnet (PM) stepper motor has a permanent magnet as a rotor, without saliencies. 2. Figure shows a simplified sketch of a PM stepper motor is shown.

The hybrid stepper motor combines the first two and has a rotor with permanent magnet and saliencies.

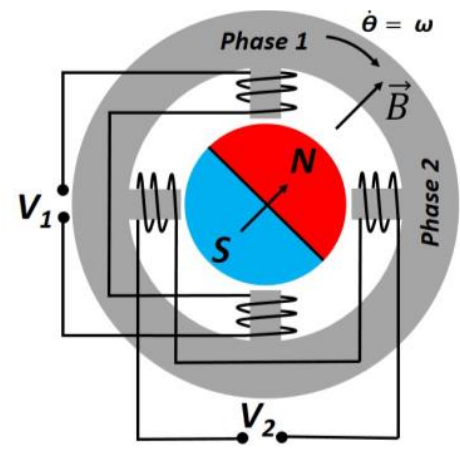

Recent Innovations in Mechatronics (RIiM) Vol 7. (2020) No 1.

DOI: $10.17667 /$ riim.2020.1/6.

2. Figure: PM stepper motor [6]

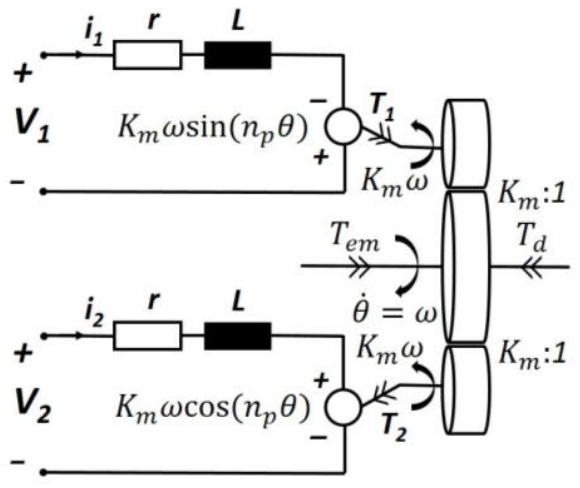

3. Figure: Equivalent circuit diagram [6]

Also, an equivalent circuit of the motor in machine variables can be created for a greater understanding of the system, shown on 3. Figure. For simplicity, we assume that there is no magnetic coupling between the phases, no magnetic saturation and that the inductance is constant, independent from rotor position.

\section{MODELING}

The Bond Graphs [7] are used to represent a dynamical system graphically, enabling us to convert the system into a state-space representation. A similarity between block diagram, signal-flow graph and Bond Graph can be observed, with the significant difference, that arrows in Bond Graphs represents bidirectional exchange of physical energy. In block diagrams and signal-flow graphs, they represent the uni-directional flow of information.

Bond Graphs (BG) are multi-energy domain, meaning that they can incorporate multiple domains seamlessly, like mechanical, electrical, hydraulic, or they can be domain neutral.

BG modelling technique was used, because the simulation is based on a BG model of a general PM stepper motor available in the professional literature about electro-mechanical system modelling [6]. Furthermore, it enables straight-forward conversion of the BG model to block diagram, which can be implemented in the LabVIEW simulation environment. The BG model of the PM stepper motor is shown on 4. Figure.
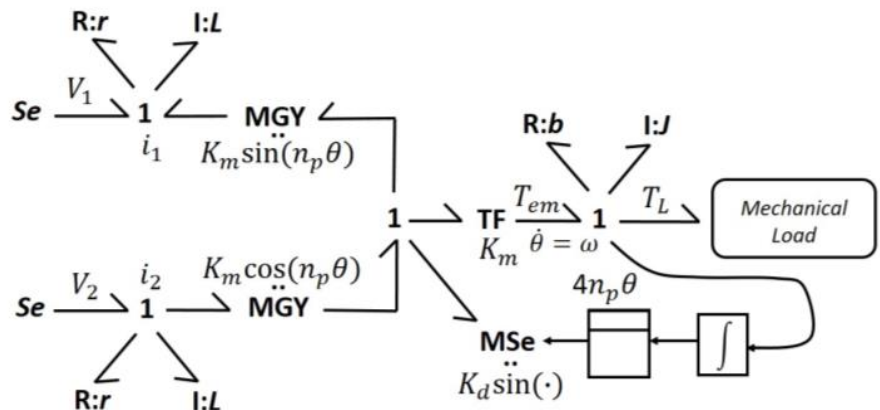

4. Figure: BG model of the PM stepper motor [6] 
In this model, the two $M G Y$ s provides a link between the electrical and mechanical subsystems in the BG. They are modulated gyrators, because the counter-electromotive force is dependent on the rotor position and pole number. An equation for each $M G Y$ is used to represent the rotation dependent change.

There is an independent torque source, the $M S e$, which is a modulated source. This torque is pure magnetically induced reluctance torque, which, even without currents flowing in the stator, tries to align the permanent magnet with the stator saliencies. This torque is called detent torque $\left(T_{d}\right)$.

To calculate this torque, the Equation (1) is used:

$$
T_{d}=K_{d} \cdot \sin \left(4 \cdot n_{p} \cdot \theta\right)
$$

The electromechanical torque $\left(T_{e m}\right)$ is dependent on the rotor angular position. The correlation between the rotor position and electromechanical torque can be calculated using the Equation (2):

$$
T_{e m}=K_{m}\left[\cos \left(n_{p} \cdot \theta\right) \cdot i_{2}-\sin \left(n_{p} \cdot \theta\right) \cdot i_{1}\right]
$$

In the Equation (2) can be observed that the electromechanical force is also depending on the intensity of the currents flowing in the motor two phases $\left(i_{1}, i_{2}\right)$.

Primary, these currents are determined by the resistance of the motor coils and the supply voltage used to power the motor. In practice, the stepper motors are driven with a current-limited constant voltage source. This current limit is determining the maximum current flow in the phases of the motor.

For simulation and in real life, for most of the cases, $i_{1}$ is equal with $i_{2}$. So this constrain will be used during the implementation of the BG in LabVIEW.

\section{IMPLEMENTING THE BOND GRAPH IN LABVIEW ENVIRONMENT}

To implement the BG of the PM stepper motor in LabVIEW, we have to expand the $\mathrm{BG}$ to a block diagram. This procedure is done by considering the rules of transformation the BG elements into block diagram.

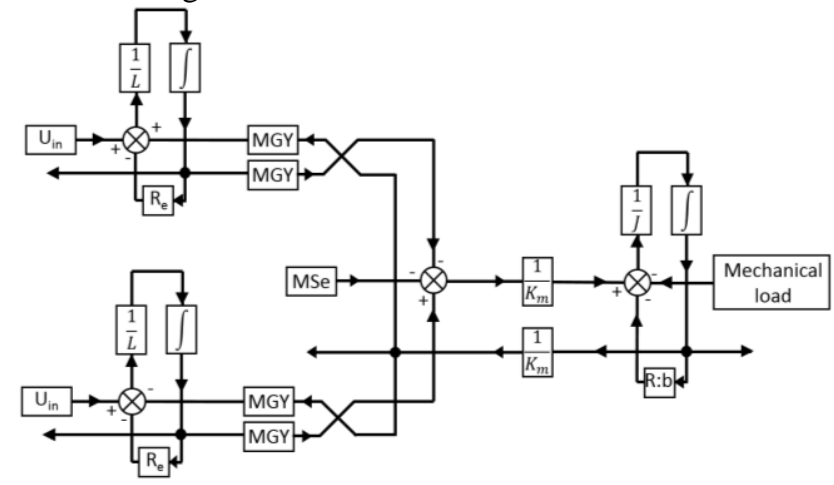

5. Figure: Block diagram created from $B G$
Recent Innovations in Mechatronics (RIiM) Vol 7. (2020) No 1.

DOI: $10.17667 /$ riim.2020.1/6.

By expanding the presented BG, the block diagram shown on 5. Figure is obtained.

These elements can be found in the LabVIEW environment, so, for simplicity, we have to redraw the diagram in the simulation environment.

Because the simulation is time-dependent, the whole block diagram was built in the "Control \& Simulation Loop" available in LabVIEW for this purpose. The elements used here are part of the "Control \& Simulation" group. Under this menu, we can find the "Simulation" submenu, which contains all the necessary modules for simulation.

The input variables for the simulation are outside of the main loop. To obtain the desired input values, basic arithmetic operations are performed on them.

The $K_{m}$ and the $K_{d}$ values are not specified in general in the datasheets of the stepper motors. Instead, we can calculate these values using the maximum current in the coils and the maximum torques.

From the Equation (1) we can obtain the value of $K_{d}$. The maximum value of the sine function is 1 . Consequently, the maximum value of the detent torque $\left(T_{d}\right)$ is when it is equal with $K_{d}$ - simplified, $K_{d}=T_{d}$.

To obtain the motor constant, used for the $M G Y$ gyrators and $1 / K_{m}$ transformer, we have to rearrange the Equation (2). First, we assume that the value of $\theta$ equals to zero. In this case, the sine function will also be zero. However, the cosine function will get the value of 1 . In this case, the Equation (3) is obtained:

$$
T_{e m}=K_{m} \cdot i_{2}
$$

By rearranging the equation, the following formula is obtained:

$$
K_{m}=\frac{T_{e m}}{i_{2}}
$$

In the Equation (4) the $K_{m}$ is the motor constant, $T_{e m}$ is the electromechanical torque and $i_{2}$ is the maximum current in each winding, which are equal in practice.

By using these equations combined with the block diagram shown on 5. Figure, the LabVIEW implementation is obtained. 


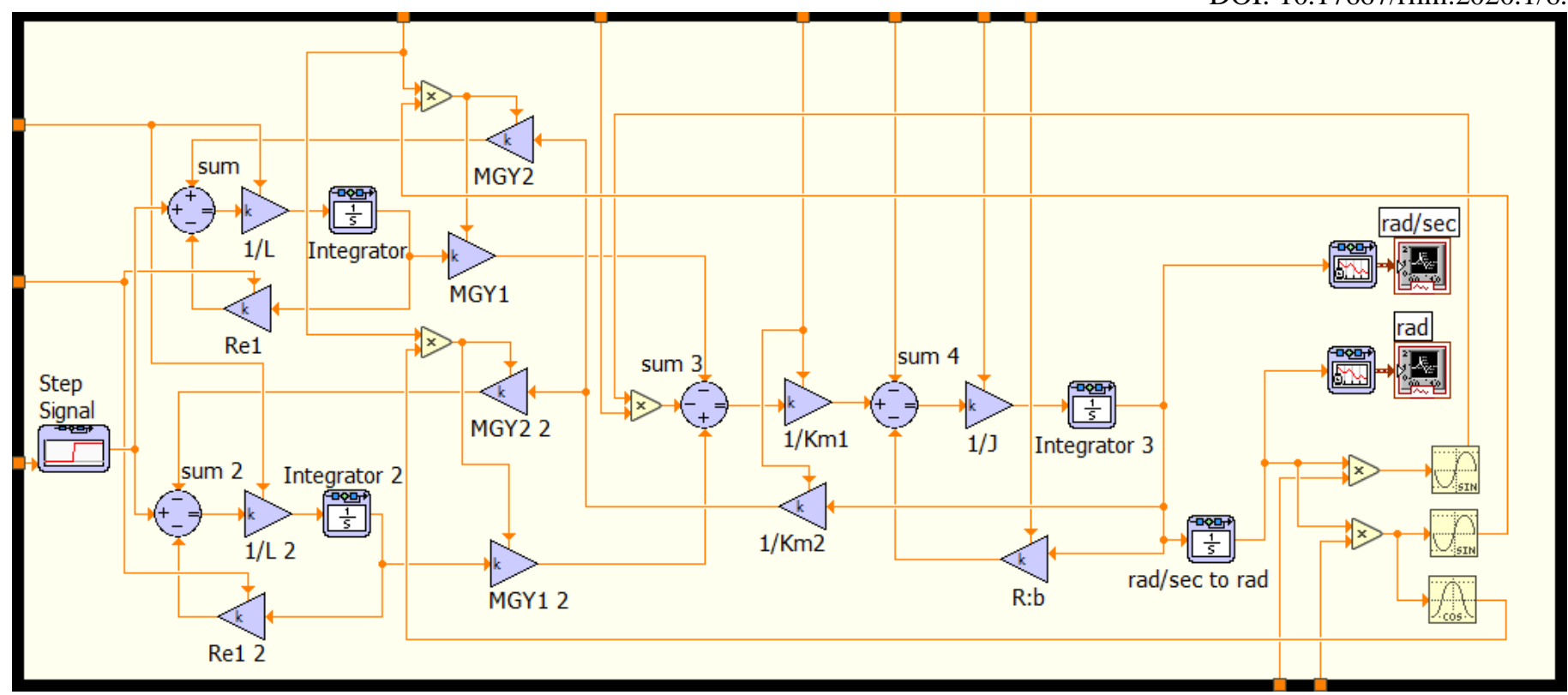

6. Figure: Simulation loop

On 6. Figure the simulation loop is shown. In this part, we can observe the implementation of the two modulated gyrators represented by the variable gains $M G Y 1, M G Y 2$, $M G Y 12$ and $M G Y 22$.

The input signal is generated by a Step Signal generator module, simulating the sudden power-on of the stepper motor.

The TR transformer part of the implemented BG is realized with the $1 / K m 1$ and $1 / K m 2$ gains.
The friction of the motor is simulated with the gain $R: b$ and the inertia torque by the $1 / J$ gain.

The $\mathrm{rad} / \mathrm{sec}$ and the $\mathrm{rad}$ graphs are used to plot the motor rotation and the angular speed in function of time. The result of the block diagram is angular velocity. To obtain angular rotation, we have to integrate the angular velocity in function of time in the simulation loop. This is done by the integrator named $\mathrm{rad} / \mathrm{sec}$ to rad.

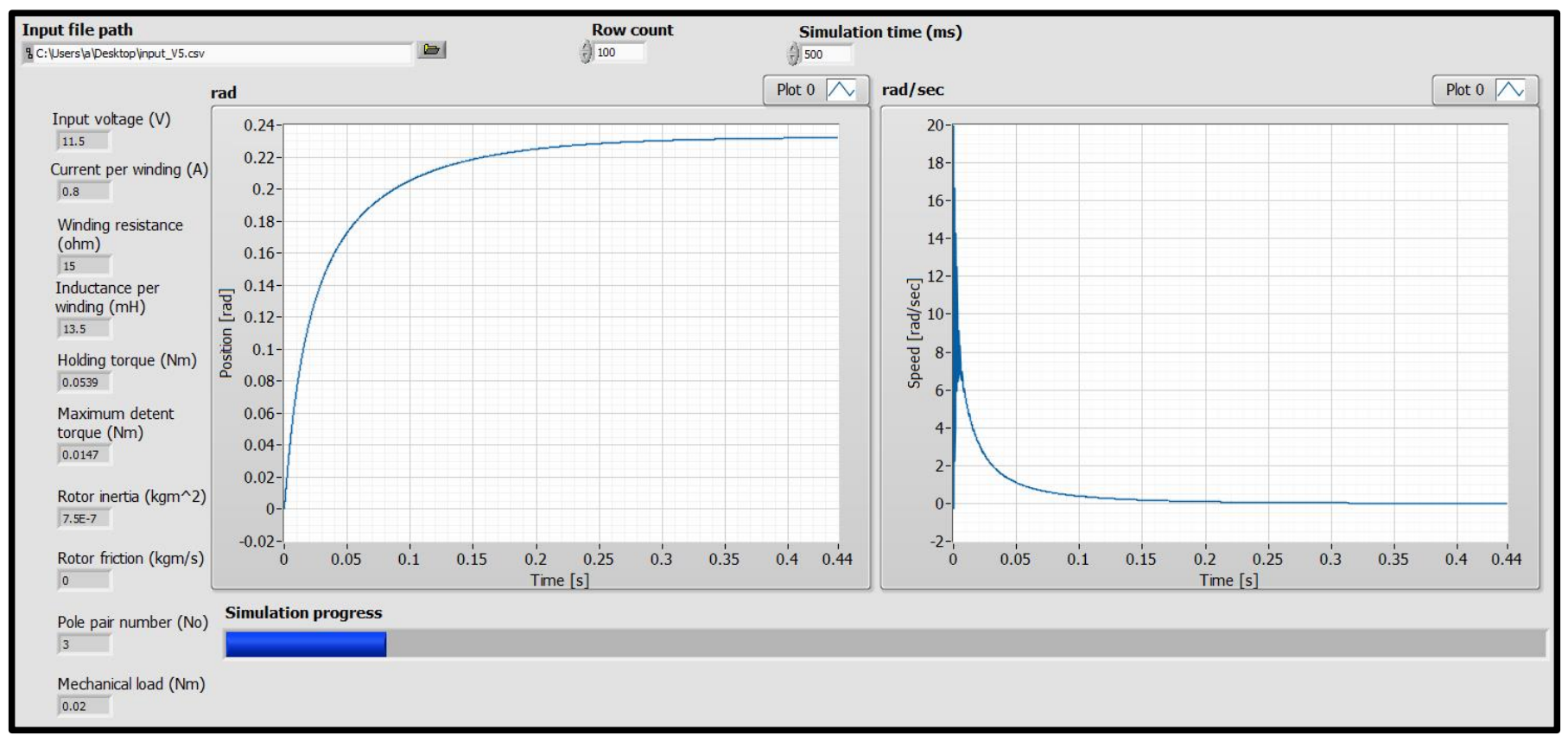

7. Figure: Simulation user interface 


\section{CONCLUSION}

\section{SIMULATION}

After implementing the model of the stepper motor, a simulation interface was created. Because manually entering the motor load parameters and simulating each scenario can be timeconsuming, an automatic data reading interface was created. The interface can read the motor parameters and the load of the motor contained in a multi-line CSV file. Each line of the file represents a different simulation scenario. These kinds of files can be processed by most spreadsheet applications, thereby enabling input file creation by the user. The results are displayed during the simulation process on the user interface - the 7 . Figure is intended to illustrate the layout arrangement of the user interface.

The repeatability of the test environment is ensured by a virtual machine set up to run the LabVIEW environment and execute the simulation. The virtual machine was simulated with VMware Workstation 15 Player, version 15.0.1. The virtual machine had the following specifications:

- CPU: Intel Core i7-4710MQ @ 2.5GHz (4 hyper-threaded cores used by the virtual machine)

- RAM: $6.5 \mathrm{~GB}$

- OS: Windows 7; 64 bit

- Storage: host computer with SSD, $560 / 540 \mathrm{MB} / \mathrm{s} \mathrm{read} /$ write rate $(150 \mathrm{~GB}$ assigned for virtual machine)

As an output, the user interface displayed graphs are saved as images. These are divided into two categories: one displaying the motor axis position in radians as a function of time and the other displaying the axis rotation speed in radians/second as a function of time.

As a demonstration, the simulation for the stepper motor described in the "Introduction" section have been carried out. The axis was loaded with $0.02 \mathrm{Nm}$ torque, and the supply voltage range changed from $5.5 \mathrm{~V}$ to $55 \mathrm{~V}$ in $0.5 \mathrm{~V}$ steps. The range means the software executes 100 simulations. The execution time for 100 different supply voltages was 36.73 seconds. This includes the saving time of the measurement data for each supply voltage.

In this way, the optimal supply voltage or the motor suitability can be determined for the specified torque. The model simulates a full step rotation of the stepper motor. In this case, a 15 degree movement. This is illustrated on the left graph of 7. Figure.

15 degrees are equal to approximately 0.261799 radians, which means that the momentary status of the simulation (7. Figure) does not ensures enough supply voltage for the motor to move a full step with $0.02 \mathrm{Nm}$ load. The $0.26 \mathrm{rad}$ positioning happens at a supply voltage of $49 \mathrm{~V}$. This means a motor phase current of $3.27 \mathrm{~A}$. This value exceeds the $0.8 \mathrm{~A}$ limit, which leads to the conclusion that the described motor in the II. section is not suitable to drive a $0.02 \mathrm{Nm}$ load.
To make sure that the designed stepper motor model represents correctly the physical stepper motor, testing a real motor and measuring its output parameters is recommended. This would include loading the motor axis with a range of different mechanical load values, while measuring the axis position and calculating its speed.

During these tests, a constant voltage to supply the motor must be provided, and a current limited motor driver must be used. These would assure that the parameters specified in the simulation are met.

If further needs arise, because the simulation was implemented in LabVIEW environment, this can be extended with new features. For example, the simulation results can be outputted in a multiline CSV format. This can be post-processed by spreadsheet applications or custom-made software.

With the data acquisition modules and functions available in LabVIEW, the measurements of a real motor and the comparison with the simulation results can be executed in a single software and in real-time. This kind of implementation opens the possibilities of future developments in motor diagnostics and preventive maintenance.

\section{ACKNOWLEDGMENT}

The work/publication is supported by the EFOP-3.6.1-16-2016-00022 project. The project is co-financed by the European Union and the European Social Fund.

\section{REFERENCES}

[1] Géza Husi, "Position Singularities and Ambiguities of the KUKA KR5 Robot," INTERNATIONAL JOURNAL of ENGINEERING TECHNOLOGIES, Vol. 1, No. 1., 2015.

[2] Kiss T., Szemes P., "Quadkopter szimulációja LabVIEW környezetben," in International Journal of Engineering and Management Science (IJEMS), DOI: 10.21791/IJEMS.2017.1.8., 2017, pp. 45-49.

[3] FAULHABER MICROMO, LLC., "Stepper Motor Technical Note: Microstepping Myths and Realities," [Online]. Available: https://www.faulhaber.com/en/support/technicalsupport/motors/tutorials/stepper-motor-tutorial-microstepping/. [Accessed 2005 2020].

[4] Modular CircuitsH-Bridges - the Basics, [Online]. Available: http://www.modularcircuits.com/blog/articles/h-bridge-secrets/hbridges-the-basics/. [Accessed 0512 2017].

[5] STEPPERONLINE, "35PM24L01 PM Stepper motor," [Online]. Available: https://www.omcstepperonline.com/download/35PM24L01.pdf. [Accessed 1005 2020].

[6] Wolfgang Borutzky, Bond Graph Modelling of Engineering Systems, London, ISBN 978-1-4419-9367-0: Springer New York Dordrecht Heidelberg.

[7] BondGraphs.com, "About Bond Graphs," [Online]. Available: https://groups.csail.mit.edu/drl/journal_club/papers/Samantaray_200 1__www.bondgraphs.com_about.pdf. [Accessed 1211 2017]. 